\title{
Estudio Comparativo de la Indicación y Tasa de Sobrevida de Materiales de Restauración Utilizados en Pacientes Pediátricos de 4 a 9 Años con Alto Riesgo de Caries
}

\author{
Comparative Study of the Indicated Survival Rate of Restorative Materials Used in \\ Pediatric Patients 4 to 9 Years of Age with High Risk of Developing Caries
}

Mónica Ceballos Casanova*; Claudia Acevedo Atala*; Alejandra Jans Muñoz* \& Claudia Atala Acevedo*

\begin{abstract}
CEBALlOS, C. M.; ACEVEDO, A. C.; JANS, M. A. \& ATALA, A. C. Estudio comparativo de la indicación y tasa de sobrevida de materiales de restauración utilizados en pacientes pediátricos de 4 a 9 años con alto riesgo de caries. Int. J. Odontostomat., 8(3):345-350, 2014.
\end{abstract}

RESUMEN: La elección del material restaurador a usar, puede ser una decisión crítica, especialmente en pacientes pediátricos de alto riesgo cariogénico y corta edad, debido a que idealmente, las restauraciones debieran acompañar al diente primario a su exfoliación natural, por lo que la indicación debe estar estrechamente ligada al riesgo cariogénico del paciente. El objetivo de este trabajo fue determinar si hay diferencias en la indicación y sobrevida de los materiales de restauración utilizados en la clínica pediátrica. Se llevo a cabo un estudio observacional retrospectivo. Se seleccionaron 60 pacientes de un universo de 369, de entre 4 y 9 años de edad, atendidos en la clínica Docente Asistencial de La Universidad de la Frontera, durante el año 2010, que cumplieron con los criterios de inclusión de esta investigación: pacientes con alto riesgo cariogénico, molares primarios con tratamiento pulpar vital, restaurados con cemento de vidrio ionómero convencional, amalgama, resina compuesta o coronas de acero pediátricas. Para el análisis estadístico de los datos se utiliza el paquete estadístico Stata 8.0, realizándose un exploratorio de los datos, test de Fisher y el estimador de Kapan Meier para el análisis de sobrevida. Fueron estudiados 150 molares primarios de 60 pacientes, con una edad promedio de 6,8 años (DE $\pm 1,5$ años). El tiempo de observación promedio fue de 10,4 meses ( $\mathrm{DE} \pm 3,7$ meses). No se observó diferencias estadísticamente significativa entre los diferentes materiales de restauración, comparados entre si, excepto entre las amalgamas y los vidrios ionómeros $(P=0,0510)$. $E l$ análisis de las tablas de sobrevida demuestra que la longevidad de las amalgamas y las coronas es similar, mientras que la restauración que presenta menor sobrevida son las realizadas con vidrio ionómeros.

PALABRAS CLAVE: riesgo cariogénico, amalgama, composites, vidrio ionómero de restauración, coronas de acero pediátricas.

\section{INTRODUCCIÓN}

En la actualidad, los odontólogos cuentan con una amplia variedad de opciones respecto al tipo de material de restauración a utilizar que hacen crítica la toma de decisiones clínicas en el tratamiento de la caries en dentición primaria (Mickenautsch \& Yengopal, 2013), especialmente en pacientes pediátricos con alto riesgo de caries y corta edad, debido a que idealmente, las restauraciones debieran acompañar al diente primario hasta u exfoliación natural (Qvist et al., 2010); sin embargo, tienen un tiempo de vida limitado.

Estudios realizados por Qvist et al., en Dinamarca demuestra que la sobrevida de las restauracio- nes es mas reducida en dentición permanente joven que en adultos (Tran \& Messer, 2003; Dodes, 2001).

En la última década, el desarrollo de materiales adhesivos, han permitido aumentar las alternativas para el tratamiento y modificar los diseños cavitarios a través de desgastes más conservadores. La mejora en las propiedades adhesivas, mecánicas y estéticas, han echo de las resinas de composites un material de elección, aunque existe el acuerdo generalizado, que se trata de un material que requiere de una técnica cuidadosa (Donly \& Garcia-Godoy, 2002; Burgess et al., 2002), mayor costo y menos duración (Opdam et al., 2007). 
Otros materiales también han experimentado mejoras, como es el caso del vidrio ionómero, cuyas características de biocompatibilidad, adherencia, liberación de fluor (Croll \& Nicholson, 2002), lo hace un material de elección en odontología pediátrica. Sin embargo características como la radiolucidez, manipulación, y baja resistencia a la compresión, limitan su indicación en superficies sometidas a carga constante (Welbury et al., 2000).

Una tendencia creciente en Europa y EEUU, a estimulado a los profesionales de esos países a restringir el uso de amalgamas de plata en niños y embarazadas, la controversia ha aumentado en el ultimo tiempo aunque hasta este momento, no existe evidencia científica que asocie este material a enfermedades sistémicas (Dodes; Osborne et al., 2002; Forss \& Widström, 2003; DeRouen et al., 2006).

Tradicionalmente se indican coronas pediátricas en dientes con amplia destrucción coronaria, porque se considera que presentan una mayor durabilidad, sin embargo son poco utilizadas por su dificultad de preparación. Randall et al. (2002) concluyen que la indicación de coronas de acero es baja, comparada con restauraciones de amalgama; establece además como posibles factores, asociados a esta indicación a la falta de entrenamiento, y la buena salud dental de los niños de la región.

Tran \& Messer publican un estudio de las opciones de elección más frecuente de materiales de restauración en niños. Encuestando a odontólogos generales y especialistas pediátricos, los resultados permitieron estimar que los odontólogos generales que atienden niños indican vidrio ionómeros en forma prioritaria, mientras que los especialistas rehabilitan con resinas de composite y coronas pediátricas de acero.

A pesar de que los investigadores se han esforzado en realizar investigaciones que permitan orientar a la comunidad odontológica, en la elección de materiales restauradores, aun la opción de selección, parece estar basada en preferencias individuales (Tran \& Messer).

Desde esta perspectiva es gravitante determinar cuáles son los materiales restauradores en niños, con mejor pronóstico clínico, con el fin de proveer una plataforma odontológica orientada a tomar decisiones más costo-efectivas cuando elegimos un material restaurador en infantes.
El propósito de esta investigación es establecer a través de la evidencia clínica, que material de restauración (coronas pediátricas, resinas fotopolimerizables, amalgamas, vidrio lonómero) presenta mayor longevidad y vida media cuando restauramos pacientes infantiles de alto riesgo cariogénico, y cual es el porcentaje de indicaciones de los cuatro tipos de restauraciones en molares deciduos, determinando a su vez la existencia de diferencias estadísticamente significativas de falla entre los diferentes tipos de restauración.

\section{MATERIAL Y MÉTODO}

Se realizó un estudio retrospectivo de tipo observacional. El universo correspondió a 369 pacientes infantiles atendidos en Clínica Docente Asistencial Universidad de La Frontera, Temuco, Chile. Durante los meses de Marzo 2010 a enero 2011 por alumnos que cursaban la asignatura Clínica Odontológica Pediátrica II $5^{\circ}$ año.

Fueron incluidos en pacientes (i) entre 4 y 9 años (8 años 11 meses), (ii) atendidos durante el periodo de Marzo 2010 a Enero 2011 y que presentaron alta integral y a lo menos 1 control de mantención, consignados en su primera atención como alto riesgo cariogénico, (iii) restaurados con coronas pediátricas, composites, ionómero y amalgamas, (iv) restaurados con resinas fotopolimerizables que abarcan mas de 3 caras del diente temporal, incluyendo restauraciones cuspídeas; (v) pacientes sometidos a terapias pulpares en piezas temporales pulpotomía, o pulpectomía y (vi) oacientes con radiografías previas a la intervención, y radiografías en el momento del alta integral (protocolo de alta integral pacientes pediátricos). Se excluyeron pacientes en periodo de recambio o exfoliación natural por la serie premolar, que presentaran dientes con movilidad (dientes restaurados), casos con ausencia de radiografías previas y de control operatorio, y pacientes con restauraciones con evidente presencia de patología periodontal, (absceso periodontal).

Todos los participantes asintieron su participación y un consentimiento informado de atención y participación en el estudio firmado por los padres o tutores.

Para efectos de esta investigación se definió alto riesgo cariogénico a aquellos niños que presentaron clínicamente, más de 4 lesiones cariosas momento del examen inicial, 4 o más momentos de azúcar, índice de O'leary 
mayor del 25\%, como criterio básicos de definición.

La realización de las acciones clínicas y la utilización de los diferentes materiales de restauración fueron realizadas bajo estrictas condiciones de aislamiento absoluto y control de fluidos, según protocolo y bajo la supervisión del equipo docente del área clínica, debidamente calibrado.

De acuerdo a los criterios establecidos se seleccionaron para la muestra 60 pacientes atendidos durante el período Marzo-Diciembre año 2009 y dados de Alta Integral, cuyas edades fluctuaban entre los 4 y 9 años, los cuales presentaban un total de 150 dientes restaurados con los materiales estudiados.

Dichos pacientes fueron citados en el periodo Abril-Julio del año 2010, para la realización de su primera mantención o control clínico, el que fue realizado por los alumnos que en el momento cursaban $\mathrm{V}$ año de Odontología, supervisado por los docentes clínicos del área, debidamente calibrados.

Se les realizó una ficha clínica diseñada especialmente para el cumplimiento de los objetivos, en la que se consignó la nueva información, haciendo énfasis en los siguientes aspectos:

- Existencia o no en cambios en la formula dentaria temporal en estudio.

- Estado de la higiene oral y condiciones periodontales. - Incidencia de lesiones cariosas.

- Condiciones clínicas y radiográficas de las restauraciones efectuadas en las piezas en estudio durante el año 2010.

- Chequeo oclusal de las restauraciones.

El total de pacientes participantes fueron evaluados por los investigadores, de acuerdo a los siguientes criterios clínicos de evaluación al control de mantención o seguimiento (se considerara falla en la restauración, e indicación de re-obturación): (i) Filtración de los márgenes, (ii) Recidiva de caries (evaluación clínica y radiográfica), (iii) Adaptación marginal deficiente, (iv) Pérdida total o parcial de la restauración (Presencia de micro filtración marginal, fracturas y /o desalojo parcial o total de las restauraciones adhesivas), (v) Fractura de remanente dentario, (vi) Dientes tratados endodonticamente sintomáticos (abscesos) y (vii) Desalojo total o parcial de corona pediátrica.
Análisis estadístico. Dada la característica retrospectiva observacional, de tipo descriptivo del estudio, los análisis estadísticos correspondientes, fueron expresados en medidas de frecuencia de presentación del evento de interés, por lo que se usaron razones y proporciones, del mismo modo se realizó un estudio de asociación y de la fuerza de la misma, usando programa stata 8.0 en la determinación de la razón de chance entre el tipo de tratamiento y la indicación de reobturación de la pieza decidua. Los análisis de sobrevida fueron efectuados usando la estimación de Kaplan Meier de la función de sobrevida

\section{RESULTADOS}

Fueron observados 150 dientes obturados con los diferentes materiales (Tabla I), pertenecientes a un total de 60 pacientes, con un promedio de edad de $6,8 \pm 1,5$ (DE) años.

Tabla I. Distribución por material de restauración en relación a la falla y no falla.

\begin{tabular}{lccccc}
\hline $\begin{array}{l}\text { Material de } \\
\text { restauración }\end{array}$ & Falla & $\%$ & $\begin{array}{c}\text { No } \\
\text { Falla }\end{array}$ & $\%$ & Total \\
\hline Coronas & 6 & 14,29 & 36 & 85,71 & 42 \\
Composites & 9 & 23,68 & 29 & 76,32 & 38 \\
Amalgamas & 4 & 11,75 & 30 & 88,24 & 34 \\
Vidrio lonómero & 11 & 30,56 & 25 & 69,44 & 36 \\
Total & 30 & 20 & 120 & 80 & 150 \\
\hline
\end{tabular}

El tiempo medio de observación estimado fue de 10,4 $\pm 3,7$ (DE) meses, presentando un mínimo de 3 y máximo 17 meses, entre el alta integral y el tiempo al primer control de mantención. La frecuencia en la indicación de los materiales de restauración se observan en la Figura 1.

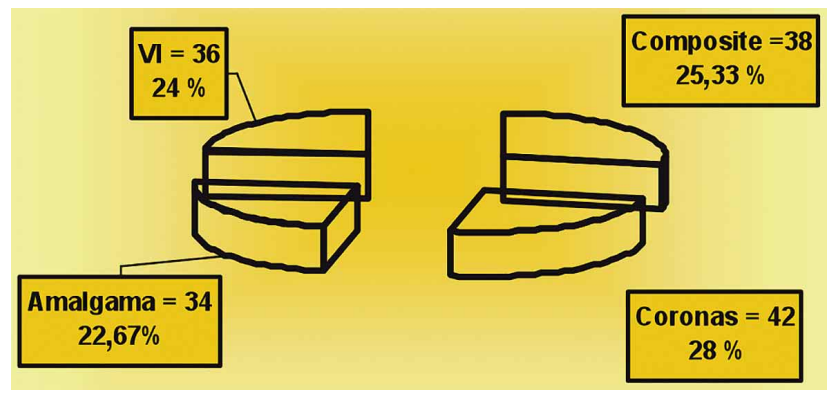

Fig. 1. Frecuencia y porcentaje de indicación de materiales de restauración en 150 molares temporales tratados endodónticamente. 
El análisis de Fischer fue realizado a través de grupos de comparación de los diferentes materiales de restauración, no se estimaron diferencias significativas, cuando se establecieron comparaciones entre amalgamas y coronas pediátricas $(P=0,3949)$, amalgama y composites $(P=0,1574)$, coronas y composites $(P=0,3070)$ ionómero y composites $(P=0,3433)$, encontrándose en el límite de la significancia estadística la establecida entre amalgamas y ionómeros $(P=0,0510)$.

El tiempo de sobrevida fue calculado según protocolo de mantención y de acuerdo al riesgo cariogénico, 3 meses entre controles. Las observaciones fueron divididas en función a los 6 tiempos de cada periodo de control $(3,6,9,12,15,17$ mes). El tiempo 17 meses fueconsiderado, pues es el último periodo observado.

La tasa de sobrevida para coronas y composites al tercer mes fue de un $100 \%$, mientras que para amalgamas y vidrio ionómero fue $91 \%$ y $97 \%$, respectivamente.

Al sexto mes, aun las coronas presentaban un $100 \%$ de sobrevida, mientras que los composites experimentaban sus primeras fallas $(6 \%)$, con una sobrevida estimada del $94 \%$, y para las amalgamas se aprecia una sobrevida del $91 \%$ (Fig. 2).

\section{DISCUSIÓN}

Los resultados obtenidos en esta muestra, no son coincidentes con la literatura al comparar coronas y amalgamas, siendo estas últimas las que presentaron mayor permanencia en el tiempo. Pensamos que esta situación se pudiera relacionar con la falta de entrenamiento de los operadores en la confección de las coronas pediátricas, coincidiendo con lo informado por Innes et al. (2007) en su revisión sistemática sobre coronas metálicas preformadas para los molares primarios cariados.

A pesar de la mejora en las propiedades de los vidrio ionómeros fotopolimerizables, estos presentan la menor sobrevida en relación a los otros materiales, aún cuando, esta sobrevida no baja del $50 \%$, considerado bueno. Esto concuerda con lo observado por Chadwick \& Evans (2007) en una revisión sistemática sobre restauraciones clase II en molares primarios, donde no se recomienda el uso de vidrio ionómero en cavidades que comprometan más de una superficie dentaria. Sin embargo en la revisión sistemática realizada por Qvist et al., se observa que el ionómero de vidrio modificado con resina y restauraciones de compómero mostraron una longevidad similar a la amalgama, mientras que las restauraciones realizadas con vidrio ionómero con-

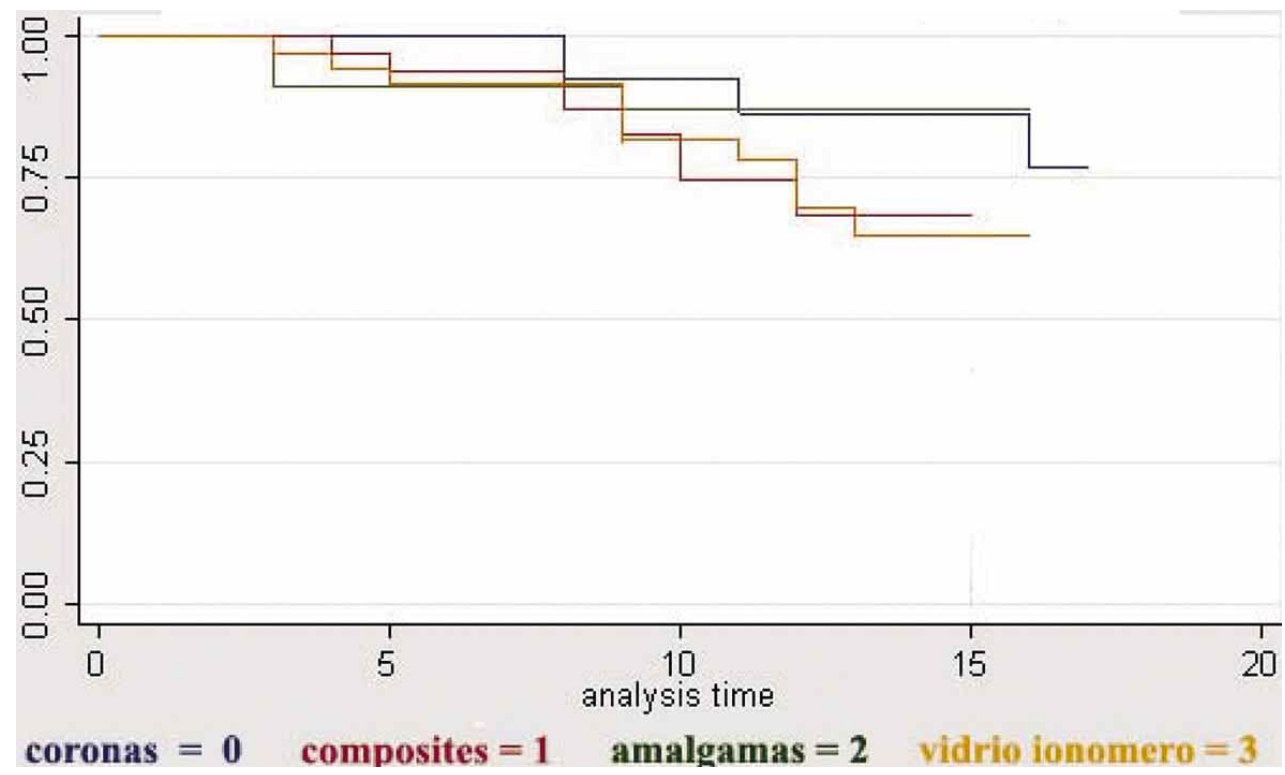

Fig. 2. Sobrevida de las diferentes restauraciones estudiadas, proyectada a los 17 meses. Se observa una tendencia que estima a las amalgamas, como la restauración de mayor sobrevida, seguida por las coronas, composites y vidrio ionómeros, respectivamente. 
vencional efectivamente evidenciaron una duración significativamente más corta.

El presente estudio arroja que los composites se proyectan como una alternativa de restauración valorable en el tiempo, misma situación encontrada por Opdam et al., considerando que éste material puede ser reparado fácilmente, sin embargo es sensible a la técnica. En este contexto Kramer et al. (2007) declara que las resinas compuestas se consideran el material restaurador que requiere mayor prolijidad en la aplicación de su técnica, aun así son ampliamente indicados (Innes et al.).

Dentro de los hallazgos encontrados se observó también que la mayor proporción de fallas en las restauraciones de composites acentuándose en el tiempo, al igual que la investigación de Opdam et al. Es necesario aumentar el tiempo de seguimiento para evaluar su comportamiento a largo plazo.
CONCLUSIONES. En el estudio se advierte que la frecuencia de indicación, es similar para los diferentes materiales de obturación cuando se trata de pacientes pediátricos entre 4 y 9 años con alto riesgo cariogénico. No existen diferencias estadísticamente significativas en la indicación de los diferentes materiales de obturación.

Las amalgamas presentan en este grupo de pacientes, a partir del noveno mes una tasa de sobrevida que se estabiliza en un $87,21 \%$, no presentando en los meses siguientes nuevos eventos o fallas. Sin embargo las restauraciones restantes continúan presentando fallas durante el tiempo de observación.

Finalmente, la sobrevida proyectada a los 17 meses, indica una tendencia que estima a las amalgamas, como la restauración de mayor sobrevida, seguida por las coronas, composites y vidrio ionómeros respectivamente (Fig. 2).

CEBALLOS, C. M.; ACEVEDO, A. C.; JANS, M. A. \& ATALA, A. C. Comparative study of the indicated survival rate of restorative materials used in pediatric patients 4 to 9 years of age with high risk of developing caries. Int. J. Odontostomat., 8(3):345-350, 2014.

ABSTRACT: The choice of restorative materials can be critical decisions, especially when it comes to high-risk pediatric patients and young cariogenic where professional commitment lies in the maintenance of the temporary works to its natural exfoliation time. So that the indication should be closely linked to the patient's caries risk. The objective of this study was to determine the survival of different restorative materials used in the pediatric clinic. We carried out a retrospective observational study, convenience sampling, 60 pediatric patients were selected from a total of 369, whose ages ranged between 4 and 9 years old and who attended the Universidad de La Frontera teaching clinic during 2010, and met inclusion and exclusion criteria of this research. Criteria included patients with high risk for caries, endodontically treated molars restored with amalgam, glass ionomer composite restoration and pediatric steel crowns. The analysis used the Stata 8.0 statistical package, Fisher exact test and survival analysis tables. We studied 150 primary molars of 60 patients, with an average age of 6.8 years (SD \pm 1.5 years). The average observation time was 10.4 months (SD \pm 3.7 months). There was no statistically significant difference between different restorative materials compared, except between amalgam and glass ionomer those found at the limit of statistical significance $(P=0.0510)$. Analysis of survival tables showed that the longevity of amalgam fillings and crowns was similar, while the restoration had lower survival rates than those made with glass ionomer.

KEY WORDS: risk cariogenic, amalgam, composites, glass ionomer restoration, pediatric steel crowns.

\section{REFERENCIAS BIBLIOGRÁFICAS}

Burgess, J. O.; Walker, R. \& Davidson, J. M. Posterior resin-based composite: review of the literature. Pediatr. Dent., 24(5):465-79, 2002.

Chadwick, B. L. \& Evans, D. J. Restoration of class II cavities in primary molar teeth with conventional and resin modified glass ionomer cements: a systematic review of the literature. Eur. Arch. Paediatr. Dent., 8(1):14-21, 2007.
Croll, T. P. \& Nicholson, J. W. Glass ionomer cements in pediatric dentistry: review of the literature. Pediatr. Dent., 24(5):423-9, 2002.

DeRouen, T. A.; Martin, M. D.; Leroux, B. G.; Townes, B. D.; Woods, J .S.; Leitão, J.; Castro-Caldas, A.; Luis, H.; Bernardo, M.; Rosenbaum, G. \& Martins, I. P. Neurobehavioral effects of dental amalgam in children: a randomized clinical trial. JAMA, 295(15):1784-92, 2006. 
Dodes, J. E. The amalgam controversy. An evidencebased analysis. J. Am. Dent. Assoc., 132(3):34856, 2001.

Donly, K. J. \& García-Godoy, F. The use of resin-based composite in children. Pediatr. Dent., 24(5):480-8, 2002.

Forss, H. \& Widström, E. The post-amalgam era: a selection of materials and their longevity in the primary and young permanent dentitions. Int. J. Paediatr. Dent., 13(3):158-64, 2003.

Innes, N. P. T.; Ricketts, D. \& Evans, D. J. P. Preformed metal crowns for decayed primary molar teeth. Cochrane Database Syst. Rev., (1), CD 005512, 2007.

Kramer, N.; Lohbauer, U. \& Frankenberger, R. Restorative materials in the primary dentition of policaries patients. Eur. Arch. Paediatr. Dent., 8(1):2935, 2007.

Mickenautsch, S. \& Yengopal, V. Validity of sealant retention as surrogate for caries prevention--a systematic review. PLoS One, 8(10):e77103, 2013.

Opdam, N. J.; Bronkhorst, E. M.; Roeters, J. M. \& Loomans, B. A. A retrospective clinical study on longevity of posterior composite and amalgam restorations. Dent. Mater., 23(1):2-8, 2007.

Osborne, J. W.; Summitt, J. B. \& Roberts, H. W. The use of dental amalgam in pediatric dentistry: review of the literature. Pediatr. Dent., 24(5):439-47, 2002.

Qvist, V.; Poulsen, A.; Teglers, P. T. \& Mjör, I. A. The longevity of different restorations in primary teeth. Int. J. Paediatr. Dent., 20(1):1-7, 2010.

Randall, R. C. Preformed metal crowns for primary and permanent molar teeth: review of the literature. Pediatr. Dent., 24(5):489-500, 2002.

Tran, L. A. \& Messer, L. B. Clinicians' choices of restorative materials for children. Aust. Dent. J., 48:(4):221-32, 2003.

Welbury, R. R.; Shaw, A. J.; Murray, J. J.; Gordon, P. H. \& McCabe, J. F. Clinical evaluation of paired compomer and glass ionomer restorations in primary molars: final results after 42 months. $\mathrm{Br}$. Dent. J., 189(2):93-7, 2000.
Dirección para Correspondencia:

Prof. Dra. Mónica Ceballos Casanova

Especialista Odontopediatria,

Mg. Epidemiología Clínica

Jefe de Clínica Odontopediatria $4^{\circ}$ Año

Universidad de La Frontera

Facultad de Odontología

Temuco

CHILE

Email: cebmonica@gmail.com

Recibido : 10-03-2014

Aceptado: $10-10-2014$ 